\title{
Percepción de la atención de enfermería y satisfacción laboral en un Hospital de Seguridad Social
}

\author{
Perception of nursing care and job satisfaction in a Social Security Hospital \\ Percepção dos cuidados de enfermagem ea satisfação no trabalho no Hospital \\ Segurança Social
}

DOI: http://dx.doi.org/10.23913/ricsh.v6i11.119

Ma. Martha Marín Laredo

Facultad de Enfermería de la Universidad Michoacana de San Nicolás de Hidalgo, México marthita_marin@yahoo.com.mx

Claudia G. Álvarez Huante

Facultad de Enfermería de la Universidad Michoacana de San Nicolás de Hidalgo, México klauz_3@hotmail.com

Josefina Valenzuela Gandarilla

Facultad de Enfermería de la Universidad Michoacana de San Nicolás de Hidalgo, México josefina_vgandarilla@yahoo.com.mx

\section{Resumen}

Las instituciones de salud en México miden la calidad de su atención a través de diversos parámetros, uno de ellos es la percepción de los profesionales de enfermería. La satisfacción laboral del personal de enfermería es un factor que los gerentes de los servicios de salud deben tomar en cuenta, ya que en gran medida determina que los usuarios hospitalizados perciban calidad en la atención.

El objetivo del presente estudio es correlacionar la calidad de la atención que perciben los usuarios hospitalizados con la satisfacción de los profesionales de enfermería del Hospital General "Vasco de Quiroga" del ISSSTE, en Morelia, estado de Michoacán. Para tal efecto se utilizó el método no experimental, descriptivo correlacional, transeccional y de campo, con un 
muestreo no probabilístico por conveniencia. Asimismo, se aplicó un cuestionario a 90 enfermeras y 70 pacientes y se utilizó el instrumento SERVQHOS- E. de Barragán y Manrique, que después de ser piloteado dio una confiabilidad de .947, mientras que para los profesionales de enfermería se aplicó el cuestionario de Font-Roja con una confiabilidad de .709 en alfa de Cronbach. Los resultados fueron los siguientes: según 44.0 \% (31) de los pacientes, el personal de enfermería manejó su equipo de trabajo (baumanómetro, estetoscopio, aspirador de secreciones, entre otros) como me lo esperaba; para $31.0 \%$ (22), mejor de lo que me esperaba, y para $30.0 \%$ (21), como no me lo esperaba. El personal de enfermería debe mostrar interés por hacer que la estancia del paciente sea lo más agradable posible. Con respecto a la satisfacción laboral con sus jefes, los profesionales de enfermería dijeron que percibían: "ambiente tenso entre directivos y operativos de enfermería"; 32.0 \% (29) dijo no estar ni de acuerdo ni en desacuerdo con ellos; $29.0 \%$ (26) dijo estar en desacuerdo y $22.0 \%$ (20) estar de acuerdo. El resto de las opciones de respuesta fueron otras. En la dimensión satisfacción por el trabajo, $71.0 \%$ (64) de las enfermeras encuestadas manifestó que se encuentra muy de acuerdo y de acuerdo con el trabajo que realiza. En conclusión, las usuarias manifestaron que el equipamiento, las señalizaciones y el estado de las habitaciones es mucho mejor de lo que esperaban, es decir, que el hospital contaba con lo necesario. Asimismo, los pacientes manifestaron que los profesionales de enfermería se interesan por hacer su estancia agradable, solucionar las problemáticas que les competen, infundirles confianza y ser amables, y que todo ello fue mejor de lo que esperaban y mисho mejor de lo que esperaban. Por otro lado, 3 de cada 10 enfermeras sufre estrés laboral y un tercio del total mencionó que el exceso de trabajo no les permite llevar a cabo sus actividades con calidad. Con respecto al ambiente laboral entre jefes y subordinados dijeron que lo perciben algo tenso, mientras que 3 de cada 10 refirieron que el salario que perciben no coincide con su carga laboral. Aunque la conclusión es que algunas enfermeras se sienten insatisfechas laboralmente, el paciente hospitalizado sigue percibiendo calidad en el cuidado que recibe.

Palabras clave: calidad, cuidado enfermero, ambiente laboral. 


\section{Abstract}

Mexico health institutions measure the quality of your care through several parameters, one of them is the perception of nursing professionals. The job satisfaction of nurses is a factor that the managers of health services should take into account, since largely determines that hospitalized users perceive quality of care.

The objective of the present study is to correlate the quality of care that perceive users hospitalized with the satisfaction of nurses in General Hospital "Vasco de Quiroga" ISSSTE, in Morelia, Michoacán. The method was used for this purpose non experimental, descriptive correlational, transactional and field, with a non-probabilistic sampling for convenience. Also, a questionnaire was applied to 90 nurses and 70 patients and used the instrument SERVQHOS-E. by Barragan and Manrique, who after being piloted gave reliability of. 947, while for nurses applied questionnaire Font-red with a reliability of.709 on Cronbach's alpha. The results were as follows: according to $44.0 \%$ (31) of the patients, the staff of nursing managed his team (sphygmomanometer, stethoscope, secretion aspirator with vacuum cleaner, among others) as expected; to $31.0 \%$ (22), better than expected, and 30.0\% (21), not expected. Nurses must show interest by making the patient's stay as pleasant as possible. With respect to job satisfaction with their bosses, nurses said they perceived: "tense atmosphere between managers and nurses"; $32.0 \%$ (29) said Neither agree nor disagree with them; $29.0 \%$ (26) said to Disagree and $22.0 \%$ (20) Agree. The rest of the response options were "other". In the dimension of satisfaction for the work, $71.0 \%$ (64) of the nurses surveyed said that they Strongly Agree and Agree in accordance with the work being done. In conclusion, users stated that equipment, signs and the State of the rooms is much better than expected, i.e., that the hospital had the necessary. In addition, patients expressed that nurses are interested in make your stay enjoyable, solve the issues that matter to them, give them confidence and be nice, and that this was better than expected and much better than expected. On the other hand, 3 of every 10 nurses suffering work-related stress and one third of the total mentioned that overwork does not allow them to carry out their activities with good quality. With regard to the working environment between bosses and subordinates said that they perceive it somehow tense, while 3 of every 10 referred to the salary they receive does not match their workload. Even if the conclusion is that some nurses feel job dissatisfaction, hospitalized continues receiving good quality care. 
Key words: quality, Nursing Care Services, work environment.

\section{Resumo}

Instituições de saúde no México medir a qualidade do seu atendimento através de vários parâmetros, um dos quais é a percepção de enfermeiros. A satisfação no trabalho do pessoal de enfermagem é um fator que os gestores de serviços de saúde devem ter em conta, como em grande parte determina que os pacientes hospitalizados percebem a qualidade do atendimento.

O objetivo deste estudo é correlacionar a qualidade do atendimento recebido pelos usuários hospitalizados com a satisfação de enfermeiros do Hospital Geral "Vasco de Quiroga" ISSSTE em Morelia, estado de Michoacán. Para este efeito foi utilizado o co transeccional e campo descritivo método experimental não, com uma amostra de conveniência probabilístico. Um questionário de 90 enfermeiras e 70 pacientes aplicada e instrumento Barragan SERVQHOS- E. Manrique e foi utilizado depois de ser pilotado deu uma fiabilidade de 0,947 , enquanto que para as enfermeiras questionário foi aplicado font-Red 709 com confiabilidade em Cronbach. Os resultados foram como se segue: 44,0\% (31 pacientes), enfermeiros dirigiu sua equipa (esfigmomanómetro, estetoscópio, aspiração de secreções, etc.) como esperado; para 31,0\% (22), melhor do que eu esperava, e 30,0\% (21) e não esperava isso. Enfermeiros devem mostrar interesse para tornar a estadia do paciente o mais agradável possível. Em relação à satisfação no trabalho com seus chefes, as enfermeiras disseram que percebida "clima tenso entre a administração e enfermagem operatório"; 32,0\% (29) disse que não era nem concordo nem discordo com eles; $29,0 \%$ (26) disse que discordou e 22,0\% (20) de acordo. As outras opções de resposta eram outros. Em satisfação no trabalho dimensão, 71,0\% (64) dos enfermeiros pesquisados declarou que foi fortemente concorda e concordam com o trabalho realizado. Em conclusão, os usuários afirmaram que o equipamento, sinais e estado dos quartos é muito melhor do que o esperado, ou seja, que o hospital precisava. Os pacientes também relataram que os enfermeiros estão interessados em fazer a sua estadia agradável, resolver os problemas que lhes dizem respeito, inspirar confiança e ser gentil, e que isso era melhor do que o esperado e muito melhor do que o esperado. Por outro lado, 3 em cada 10 enfermeiros sofrem de stress relacionado com o trabalho e um terço do total mencionado que o excesso de trabalho não permitir que eles realizem suas atividades com qualidade. Em relação ao ambiente de trabalho entre chefes e subordinados eles disseram que perceberam um pouco tenso, enquanto 3 dos 10 informou que o 
salário que eles recebem não corresponde à sua carga de trabalho. Embora a conclusão é que alguns enfermeiros estão insatisfeitos com seus empregos, em regime de internamento ainda percebido a qualidade do atendimento que você recebe.

Palavras-chave: qualidade, de cuidados de enfermagem, ambiente de trabalho.

Fecha Recepción: Julio 2016 Fecha Aceptación: Diciembre 2016

\section{Introducción}

El personal de enfermería representa más del $50.0 \%$ de los recursos humanos de las instituciones de salud, por lo que conocer los factores que inciden en su desempeño laboral es prioritario. De esa manera, las unidades hospitalarias pueden ejecutar estrategias para el logro de la satisfacción laboral y generar una óptima actitud de servicio que se refleje en el ambiente laboral y en la calidad que recibe el usuario hospitalizado (García, M. et al., 2007).

La enfermería todavía muestra algunas deficiencias en su objetivo de brindar atención de calidad y bienestar.

\section{Calidad de la atención en salud}

El concepto de calidad en el área de la salud es bastante general, abstracto y de doble perfil: objetivo y subjetivo. Sus múltiples dimensiones reciben influencia de factores culturales y de las personas que ostentan el poder.

Avedis Donabedian definió en 1980 a la calidad asistencial como "aquella que se espera que pueda proporcionar al usuario el máximo y más completo bienestar, después de valorar el balance de ganancias y pérdidas que pueden acompañar el proceso en todas sus partes". En 1989, la International Organization for Standarization (ISO) definió a la calidad como: "El grado en que las características de un producto o servicio cumplen los objetivos para los que fue creado" (citados en Net, À y Suñol, R. s/f). Por su parte, Donabedian opina que la calidad asistencial es una propiedad de la atención médica que puede alcanzarse en grados diversos. 


\section{Componentes de la calidad asistencial}

Según Donabedian, son tres los componentes de la calidad asistencial que se deben tomar en cuenta:

1. El componente técnico: es la aplicación de la ciencia y de la tecnología en el manejo de los problemas de la persona, de manera que rinda el máximo beneficio sin aumentar con ello sus riesgos.

2. El componente interpersonal: se basa en el postulado de que la relación entre las personas debe seguir las normas y valores sociales que gobiernan la interacción de los individuos en general. Estas normas están modificadas en parte por los dictados éticos de los profesionales, así como por las expectativas y las aspiraciones de cada individuo.

3. Los aspectos de confort: son todos aquellos elementos del entorno del usuario que le proporcionan una atención mas confortable (Net, À y Suñol, R, s.f).

A partir de este análisis, numerosos autores han postulado las dimensiones que abarca la calidad, siendo una de ellas la que H. Palmer refiere:

1. Efectividad: capacidad de un determinado procedimiento o tratamiento en su aplicación real para obtener los objetivos propuestos.

2. Eficiencia: prestación de un máximo de unidades comparables de cuidados por unidad de recursos utilizados.

3. Accesibilidad: facilidad con que puede obtenerse la asistencia frente a barreras económicas, organizativas, culturales, entre otras.

4. Aceptabilidad: grado de satisfacción de los usuarios con respecto a la atención.

5. Competencia profesional: capacidad del profesional para la mejor utilización de sus conocimientos a fin de proporcionar cuidados y satisfacción a los usuarios (Palmer, H. citado en Net, À y Suñol, R, s.f). 
En 1993, la oficina regional de la Organización Mundial de la Salud (OMS) para Europa, junto con las autoridades sanitarias danesas, formuló una propuesta en favor del mejoramiento continuo de la calidad en la atención sanitaria, la cual cubría los siguientes aspectos:

1. Una definición de la calidad y de los principales componentes de la atención sanitaria.

2. La identificación de los principales protagonistas, de las responsabilidades y de las actividades en todos los niveles del sistema de atencion sanitaria de un país.

3. La esencia en el mejoramiento continuo de la calidad, es decir, utilizar los mejores resultados de la atención a partir de:

a) Las experiencias de los pacientes.

b) Las actividades de nivel local, que deben formar parte del trabajo cotidiano de la totalidad del personal (Racoveanu, N. T. y Staehr, Kirsten, 1995).

Por su parte, Vanormalingen (1996) refiere que la calidad de la atención médica tiene cinco elementos principales: a) excelencia profesional, b) uso eficiente de los recursos, c) mínimo riesgo para el paciente, d) alto grado de satisfacción y, e) impacto final en la salud. Sin embargo, la calidad de la salud no se reduce a ellos, pues necesariamente implica la integración de elementos de carácter técnico y también de procesos objetivos y subjetivos; todo esto en su conjunto trae como resultado la satisfacción de los usuarios y la eficiencia de la institución de salud (Massip, C et al., 2008).

Se entiende por buena calidad la atención o el servicio que atiende los requisitos establecidos y, dados los conocimientos y recursos disponibles, que satisface las aspiraciones de obtener el máximo de beneficios con el mínimo de riesgos para la salud y bienestar de los pacientes. Por consiguiente, una atencion sanitaria de buena calidad se caracteriza por lo siguiente: un alto grado de competencia profesional; la eficiencia en la utilizacion de los recursos; el riesgo mínimo para los pacientes; la satisfacción de los usuarios; y un efecto favorable en la salud (Racoveanu, N. T. y Staehr, Kirsten, 2013, p. 159). 
Estos elementos se cumplen si los profesionales de la salud los conocen y los utilizan de forma constructiva en las siguientes esferas:

La estructura, o sea la forma en que está organizada la atención, en lo que se refiere a las condiciones económicas, la gestión, el personal, el equipo, las instalaciones y los sistemas de información.

El proceso, que comprende las aptitudes desplegadas en el suministro de información y en la prestación de servicios de prevención, diagnóstico, tratamiento y rehabilitación de los pacientes.

Los resultados, es decir, los efectos de la atención en la salud y bienestar de los pacientes, el grado en que quedan satisfechos y la eficacia en la utilización de los recursos.

Por tanto, la calidad de la asistencia sanitaria la definimos en los siguientes términos: calidad asistencial significa dar la respuesta más adecuada a las necesidades y expectativas de los usuarios de los servicios de salud, con los recursos humanos y materiales disponibles y el nivel de desarrollo científico actual, para lograr el máximo grado de desarrollo posible de satisfacción, tanto para el usuario como para los profesionales y al costo más razonable (Moreno, A., 2005).

\section{Calidad de la atención de enfermería}

Existen numerosos estudios con resultados negativos en cuanto a la mala calidad de la atención en enfermería y la satisfacción de los pacientes. La mayoría afirma que la atención en enfermería tiene significativas deficiencias y, por tanto, que debe mejorar.

Los actuales elementos de los que se dispone para brindar cuidados de enfermería con calidad son insuficientes, ya que siguen presentándose inconsistencias tales como: inadecuada entrega y recibo de turno, discontinuidad del cuidado, escasa atención a las necesidades de salud de los pacientes, aumento en las tasas de eventos adversos atribuidos a enfermería, falta de comunicación entre el equipo interdisciplinario, escasez de información y orientación al paciente sobre los procedimientos a realizar, falta de atención al llamado, e incluso que el paciente no llega a conocer el servicio en el que se encuentra hospitalizado y reconocer al personal de enfermería que lo cuida debido a que este no se presenta con él. Todo lo anterior termina por generar insatisfacción (Borré, Y. M. y Vega, Y., 2014). 
El quehacer de la enfermería en las instituciones de salud es el cuidado directo, continuo y permanente de personas en situación de enfermedad. Por tanto, brindar calidad es un proceso cuyo objetivo es alcanzar un alto nivel de excelencia en la prestación del cuidado y la satisfacción de los usuarios. Para lograrlo es necesario tener en cuenta que la presencia, la permanencia, la continuidad y la contingencia son condiciones específicas y distintivas de dicho cuidado, que se constituyen en insumos centrales de la atención a la salud, con la cual es posible proveer protección, confort y apoyo que no amenacen la integridad de las personas bajo cuidado (Lenis,V., Manrique, A. y Fred, G., 2015).

La calidad de la atención se ha convertido en un área de interés para los investigadores y gerentes de empresas de la salud debido a que impacta en el desempeño y los costos de los negocios. Cuando se superan las necesidades de los usuarios y sus expectativas, aumenta el prestigio de las instituciones y la calidad de los servicios prestados; el contacto con el personal de enfermería debe ser permanente y progresivo al ser un punto clave en la evaluación asistencial y, en consecuencia, en la calidad de la atención (Barragán J.A., Pedraza Y.D., Rincón M.R., 2013).

\section{Satisfacción laboral}

Muchas enfermeras trabajan en ambientes muy complejos donde brindan atención a pacientes en estado crítico, enfrentando diariamente conflictos emocionales como sufrimiento y muerte, atención de patologías infectocontagiosas, temor a pincharse y contagiarse de VIH o hepatitis C, así como conflictos laborales, por ejemplo, sobrecarga de trabajo, falta de equipo y/ o materiales, y áreas físicas inadecuadas. En algunos servicios hospitalarios los profesionales de enfermería se enfrentan a situaciones altamente estresantes debido a que la vida del paciente se encuentra en constante riesgo. La enfermería es una profesión particularmente estresante, ya que afecta la salud y la satisfacción laboral de sus profesionales, e implica mucha responsabilidad, así como falta de autonomía y autoridad a la hora de tomar decisiones. Como profesión, debe adaptarse a los rápidos cambios tecnológicos, la disminución de las retribuciones y estímulos de distinta clase, las condiciones físicas externas inadecuadas, las malas relaciones interpersonales y la superposición familia-trabajo. 
Estudiar la satisfacción laboral implica un aspecto complejo que no sólo abarca actividades innatas del profesional de enfermería, como el género, la edad o el grado de estudios, sino también del entorno laboral, como el reconocimiento, el trabajo en grupo o las posibilidades de ascenso (Tapia, H. et al., 2009, pp. 21-25).

Frederick Herzberg realizó estudios para determinar cuáles eran los factores en el entorno de trabajo de un empleado originados por la satisfacción o la insatisfacción, encontrando que los factores que causan la satisfacción en el trabajo eran diferentes a los causantes de la insatisfacción. Para poder explicarlo desarrolló la teoría de la motivación de higiene, donde llamó "motivadores" a los factores satisfactorios y de "higiene" a los insatisfactorios. Formuló la teoría de los dos factores para explicar mejor el comportamiento de las personas en el trabajo y planteó la existencia de dos factores que orientan el comportamiento de las personas (De Andrade et al., 2012). Son muchos los autores que definen la satisfacción laboral como un constructo multidimensional y que señalan que las siguientes dimensiones: la satisfacción con la supervisión, la empresa, los compañeros de trabajo, las condiciones de trabajo, el progreso en la carrera, las perspectivas de promoción, el sueldo, los subordinados, la estabilidad en el trabajo, el tipo de trabajo, la cantidad de trabajo, el desarrollo personal, la satisfacción extrínseca general y la satisfacción intrínseca general (Bravo, Peiró y Rodríguez, en Berrios, P. et al., 2006, p. 5).

Para un primer grupo de autores, la satisfacción laboral refiere un estado emocional, sentimientos o respuestas afectivas.

Para Muchinsky (2000, p. 238) es "el grado de placer que el empleado obtiene de su trabajo". De esta forma, afirman que un trabajador se siente satisfecho con su trabajo cuando a partir del mismo experimenta sentimientos de bienestar, placer o felicidad. Por su parte, Bravo et al. (2002) la definen como "una actitud o conjunto de actitudes desarrolladas por la persona hacia su situación de trabajo" (Navarro, E. et al., 2010).

Existe evidencia suficiente en la literatura que muestra que las instituciones del más alto nivel de calidad en los servicios de salud se denominan magnéticas porque han obtenido la certificación o acreditación desde esta perspectiva, logran el reconocimiento social y fomentan la satisfacción del profesional de enfermería (Hernández, A. y Zárate R.M., 2011, pp. 25-32). 


\section{Objetivos general}

Correlacionar la calidad de la atención percibida en los usuarios hospitalizados con la satisfacción de los profesionales de enfermería del Hospital General "Vasco de Quiroga" del ISSSTE, en Morelia, estado de Michoacán.

\section{Objetivos específicos}

Relacionar los factores sociodemográficos e institucionales de los pacientes: edad, género, estado civil, nivel de escolaridad, servicio, turno y días de estancia hospitalaría, con la calidad de la atención de enfermería que perciben.

Contrastar los factores sociodemográficos y laborales del personal de enfermería: edad, género, estado civil, preparación académica, institución donde labora, turno, categoría laboral, antigüedad en la institución y servicio asignado, con la satisfación laboral de los profesionales de enfermería.

\section{Método}

Investigación de tipo no experimental, descriptivo, comparativo, transeccional y de campo. Se utilizó muestreo no probabilístico por conveniencia. Previo consentimiento informado se un cuestionario a 90 enfermeras y a 70 pacientes del Hospital General "Vasco de Quiroga" del ISSSTE, en Morelia, estado de Michoacán.

Para recolectar la información se utilizó la técnica de la encuesta y como instrumento el cuestionario de Font-Roja de satisfacción laboral (Núñez E., Estéves G., Hernández P. y Marrero C., 2007), el cual fue modificado para los fines de la presente investigación. El instrumento modificado se estructuró con nuevas dimensiones: satisfacción en el trabajo, tensión relacionada con el trabajo, competencia profesional, presión del trabajo, mejorar a nivel profesional y reconocimiento por el trabajo, relación interpersonal con los jefes, características extrínsecas de estatus, monotonía laboral e influencia del entorno. Fueron 28 ítems, de los cuales se eliminaron cinco reactivos por su baja saturación quedando 23 ítems con escalamiento tipo Likert, que va de 1 (menor grado de satisfacción) a 5 (mayor grado de satisfacción). Al cuestionario de Font- Roja que se utilizó en este trabajo se adicionaron los factores sociodemográficos y laborales de las enfermeras, como: edad, género, estado civil, preparación académica, institución donde labora, turno, categoría laboral, antigüedad en la institución y servicio asignado. Se efectuó prueba piloto 
con personal de enfermería de un hospital que reunía características similares al objeto de estudio. La confiabilidad de alfa de Cronbach fue de .709.

Para identificar la calidad de la atención de enfermería percibida por los usuarios hospitalizados se utilizó el instrumento de Barragán y Manrique (2010), quienes realizaron un ajuste al cuestionario SERVQHOS original que denominaron SERVQHOS- E., el cual se utiliza específicamente para evaluar la calidad percibida con respecto al cuidado de enfermería. Dicho instrumento cuenta con una confiabilidad de 0.96 en la medición del constructo de calidad percibida en la atención de cuidados enfermeros, en especial en los aspectos relacionados con el trato, la confianza, la preparación del personal y el cuidado individualizado. El instrumento se piloteó en un hospital con características similares al objeto de estudio, con una confiabilidad de alfa de Cronbach de .947 y dos dimensiones: calidad objetiva del servicio de enfermería con cuatro reactivos (tecnología de los equipos, la apariencia del personal de enfermería, señalizaciones para orientarse y el estado de las habitaciones) y la calidad subjetiva del servicio de enfermería con 12 reactivos con escalamiento tipo Likert. Al cuestionario se agregaron los factores sociodemográficos de los pacientes y de la institución: edad, género, estado civil, nivel de escolaridad, ocupación, servicio, turno y días de estancia hospitalaria. 


\section{Resultados}

Tabla 1. Variables sociodemográficas de los pacientes.

\begin{tabular}{|c|c|c|}
\hline V A R I A B L E & $\mathrm{F}$ & $(\%)$ \\
\hline \multicolumn{3}{|l|}{ Edad (años) } \\
\hline $20-25$ & 4 & 5.7 \\
\hline $26-30$ & 11 & 15.7 \\
\hline $31-35$ & 14 & 20.0 \\
\hline $36-40$ & 13 & 18.6 \\
\hline 41 y más & 28 & 40.0 \\
\hline \multicolumn{3}{|l|}{ Estado civil } \\
\hline Soltero(a) & 10 & 14.3 \\
\hline Casado(a) & 36 & 51.4 \\
\hline Viudo(a) & 11 & 15.7 \\
\hline Divorciado(a) & 6 & 8.6 \\
\hline Unión libre & 7 & 10.0 \\
\hline Escolaridad & 26 & 37.1 \\
\hline Primaria & 11 & 15.7 \\
\hline Secundaria & 7 & 10.0 \\
\hline Preparatoria & 5 & 7.1 \\
\hline Nivel técnico & 18 & 25.7 \\
\hline Licenciatura & $3(4.3)$ & 4.3 \\
\hline Otro & & \\
\hline Turno & & \\
\hline
\end{tabular}




\begin{tabular}{|c|c|c|}
\hline Matutino & $\mathbf{5 1}$ & $\mathbf{7 2 . 9}$ \\
Vespertino & 16 & 22.9 \\
Nocturno & 1 & 1.4 \\
Jornada acumulada & 2 & 2.9 \\
& & \\
\hline
\end{tabular}

Fuente: 70 cuestionarios aplicados a pacientes hospitalizados

Tabla 2. Variables institucionales relacionadas con los pacientes hospitalizados.

\begin{tabular}{|c|c|c|}
\hline VARIABLE & $\mathrm{F}$ & $\%$ \\
\hline Turno & & \\
\hline Matutino & 51 & 72.9 \\
\hline Vespertino & 16 & 22.9 \\
\hline Nocturno & 1 & 1.4 \\
\hline Jornada acumulada & 2 & 2.9 \\
\hline \multicolumn{3}{|l|}{ Días de estancia hospitalaria } \\
\hline $1-3$ & 41 & 58.6 \\
\hline $4-6$ & 20 & 28.6 \\
\hline $7-9$ & 3 & 4.3 \\
\hline 10 y más & & 8.6 \\
\hline & 6 & \\
\hline
\end{tabular}

Fuente: 70 cuestionarios aplicados a pacientes hospitalizados 
Los principales resultados sobre la calidad percibida en la atención de enfermería son:

\section{Aspectos tangibles}

Con respecto a la manera como el usuario hospitalizado considera el equipo (baumanómetro, estetoscopio, aspirador de secreciones, etcétera) que utiliza el personal de enfermería para realizar su trabajo, $44.0 \%$ (31 pacientes) manifestó que fue como me lo esperaba, $23.0 \%$ (16 pacientes) que fue mejor de lo que esperaba y $21.4 \%$ (15 pacientes) que fue mucho mejor de lo que esperaba; el porcentaje restante se ubicó en otros rubros (gráfica 1).

\section{Gráfica 1}

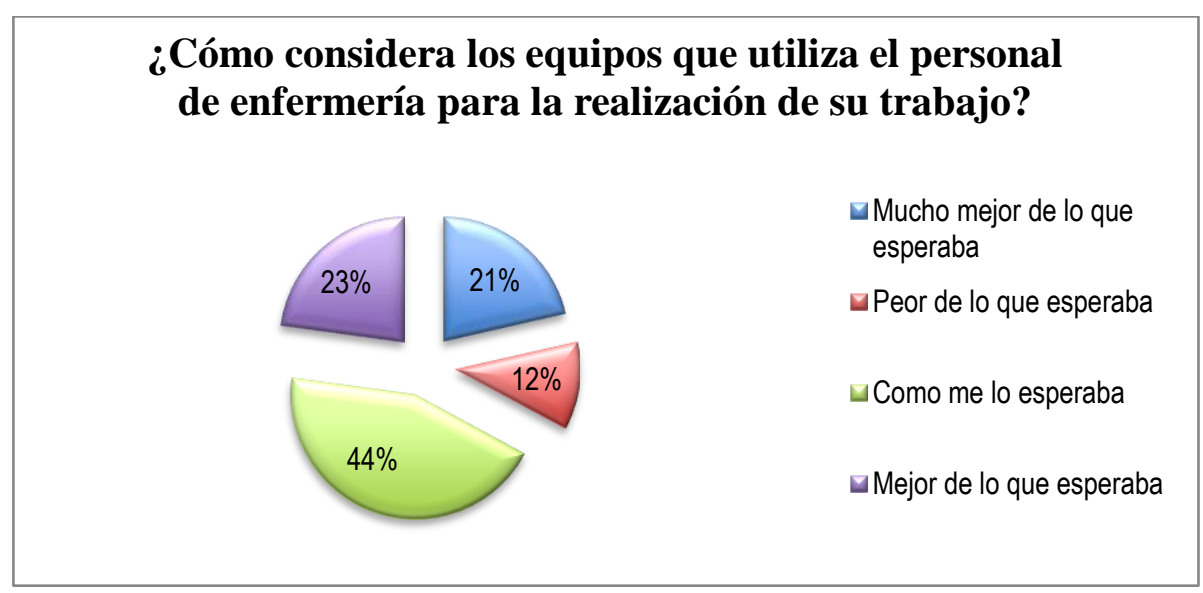

Fuente: 70 cuestionarios aplicados a pacientes hospitalizados

Con respecto a la manera como el paciente considera las señalizaciones que dispuso el hospital para orientarse (escalera, baños, salida de emergencia, laboratorio, rayos X, etcétera), 57.0 \% (40 pacientes) respondió como me lo esperaba, $19.0 \%$ (13 pacientes) dijo mejor de lo que esperaba y $17.0 \%$ (12 pacientes) mucho mejor de lo que esperaba (gráfica 2). 


\section{Gráfica 2}

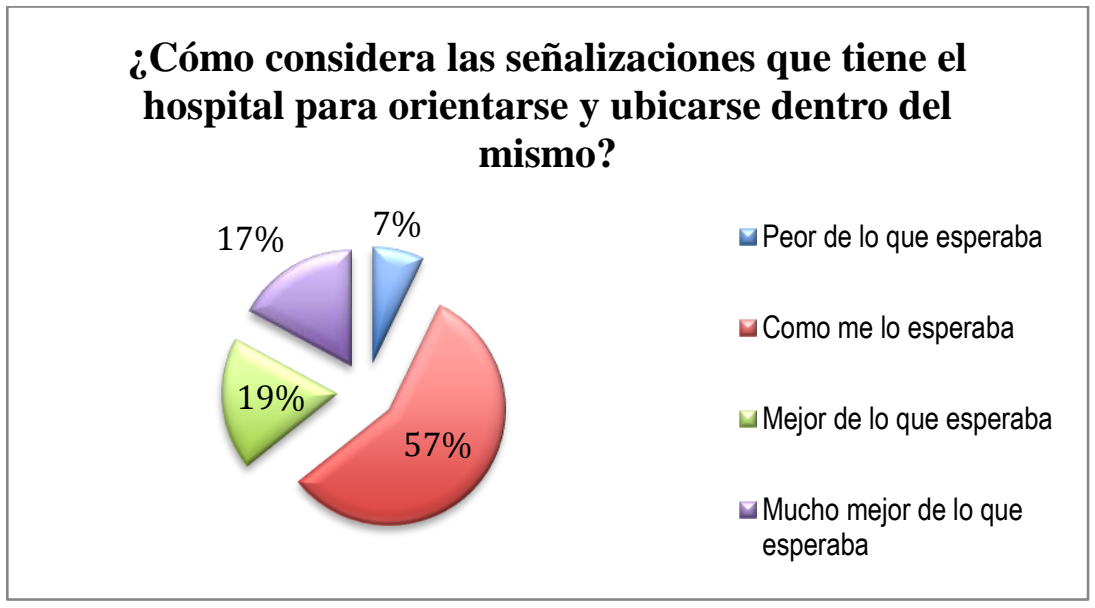

Fuente: 70 cuestionarios aplicados a pacientes hospitalizados

En la dimensión calidad subjetiva del servicio de enfermería los resultados fueron: el personal de enfermería se interesa porque dentro de lo posible su estancia sea agradable. Al respecto, $31.0 \%$ (22) comentó mejor de lo que esperaba, 30.0 \% (21) dijo como me lo esperaba y $27.0 \%$ (19) mucho mejor de lo que esperaba. El restante eligió otras opciones de respuesta (gráfica 3).

\section{Gráfica 3}

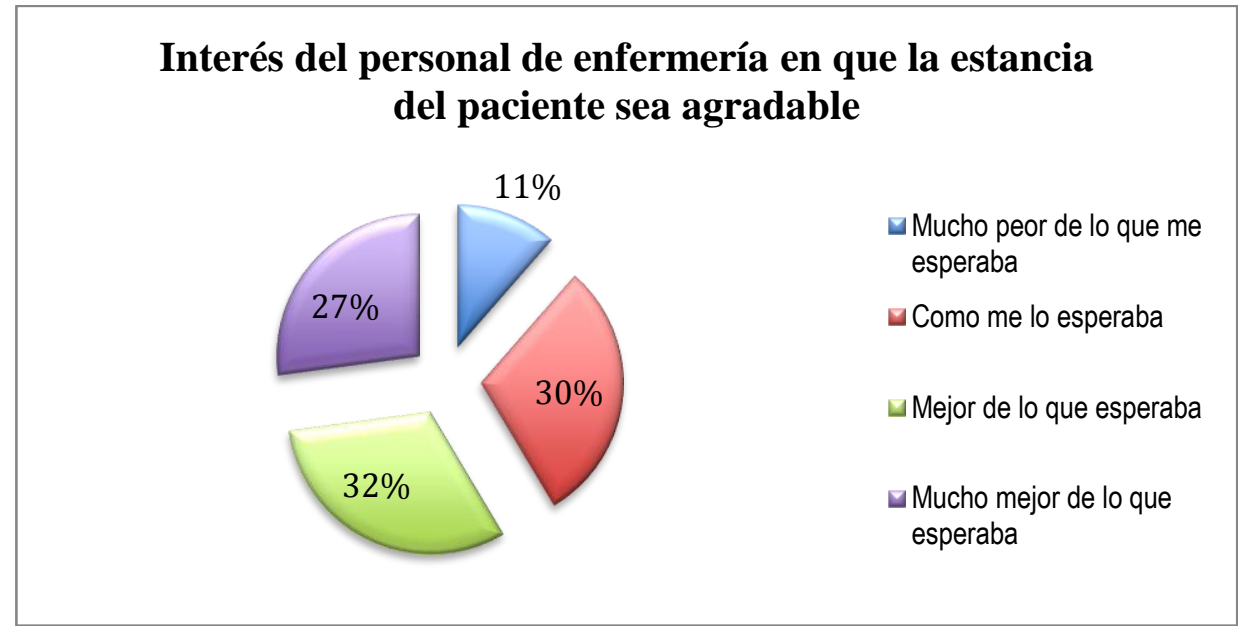

Fuente: 70 cuestionarios aplicados a pacientes hospitalizados 
En cuanto a lo que considera el paciente sobre la preparación académica del personal de enfermería para realizar su trabajo, $37.0 \%$ (26 pacientes) dijo que fue mucho mejor de lo que esperaba, $30.0 \%$ (21 pacientes) que fue como me lo esperaba, mientras que el porcentaje restante eligió otras opciones (gráfica 4).

\section{Gráfica 4}

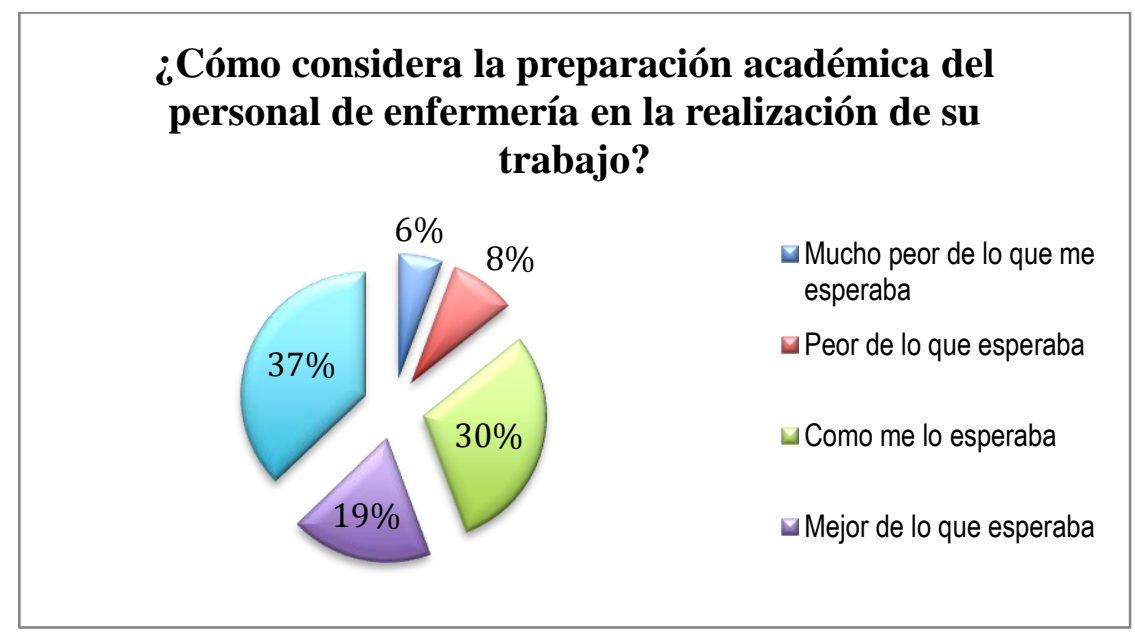

Fuente: 70 cuestionarios aplicados a pacientes hospitalizados

Con respecto al trato en enfermería que se brinda al paciente durante su estancia hospitalaria, 33.0 \% (23) respondió mucho mejor de lo que esperaba, 54.0 \% (38) comentó como me lo esperaba, y mejor de lo que esperaba, mientras que el resto eligió otras opciones (gráfica 5).

\section{Gráfica 5}

¿Cómo considera el trato que brinda enfermería al paciente durante su estancia hospitalaria?

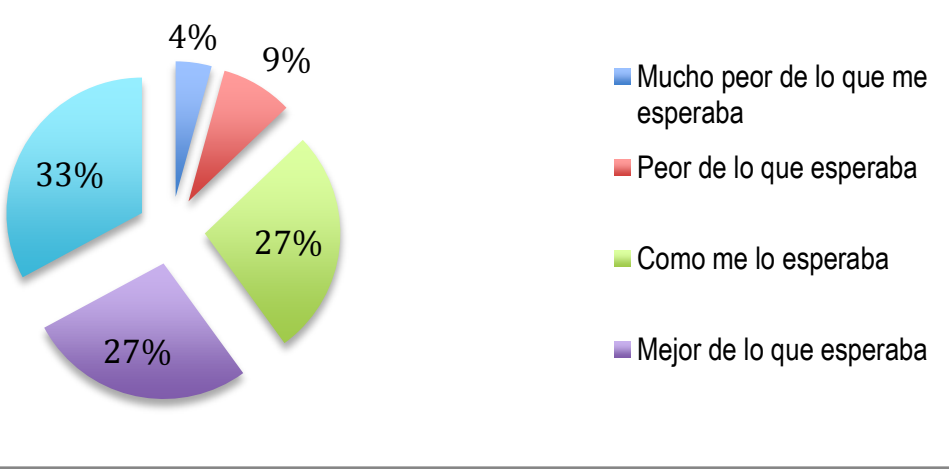




\section{Perfil sociodemográfico y laboral de los profesionales de enfermería}

En cuanto a la edad del personal de enfermería, $18.0 \%$ (16) dijo que tiene entre 35 y 39 años, y $41.0 \%$ (37) que tiene más de 40 años de edad, el resto fue ubicado en otros grupos (gráfica 6).

\section{Gráfica 6}

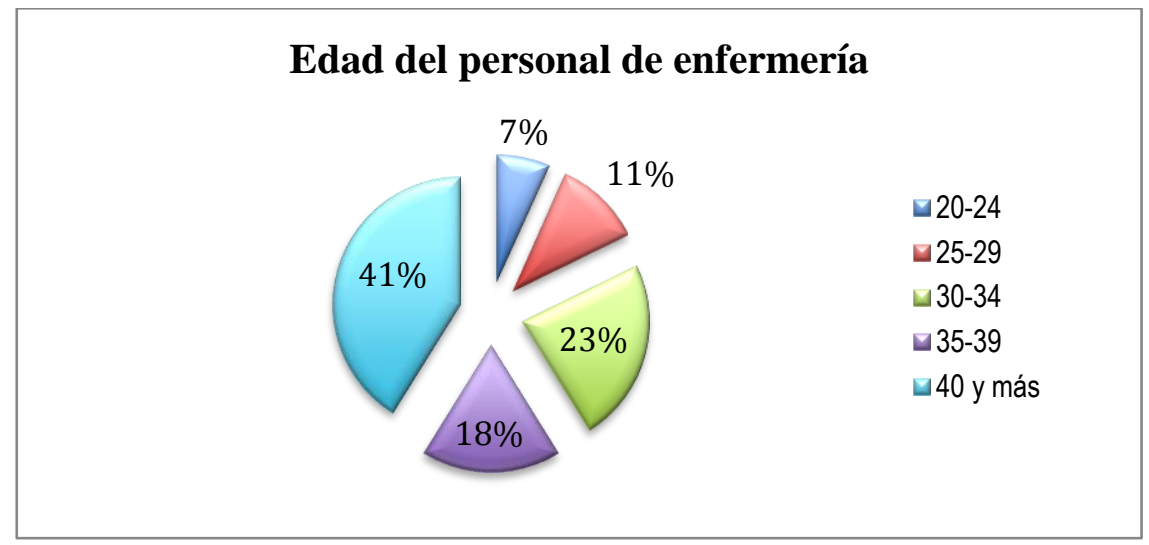

Fuente: 90 cuestionarios aplicados al personal de enfermería.

En la antigüedad del personal de enfermería en la institución de salud se observa que 55.0 \% (49) tiene 15 años de antigüedad y 45. 0 \% (41) más de 16 años de antigüedad (gráfica 7).

\section{Gráfica 7}

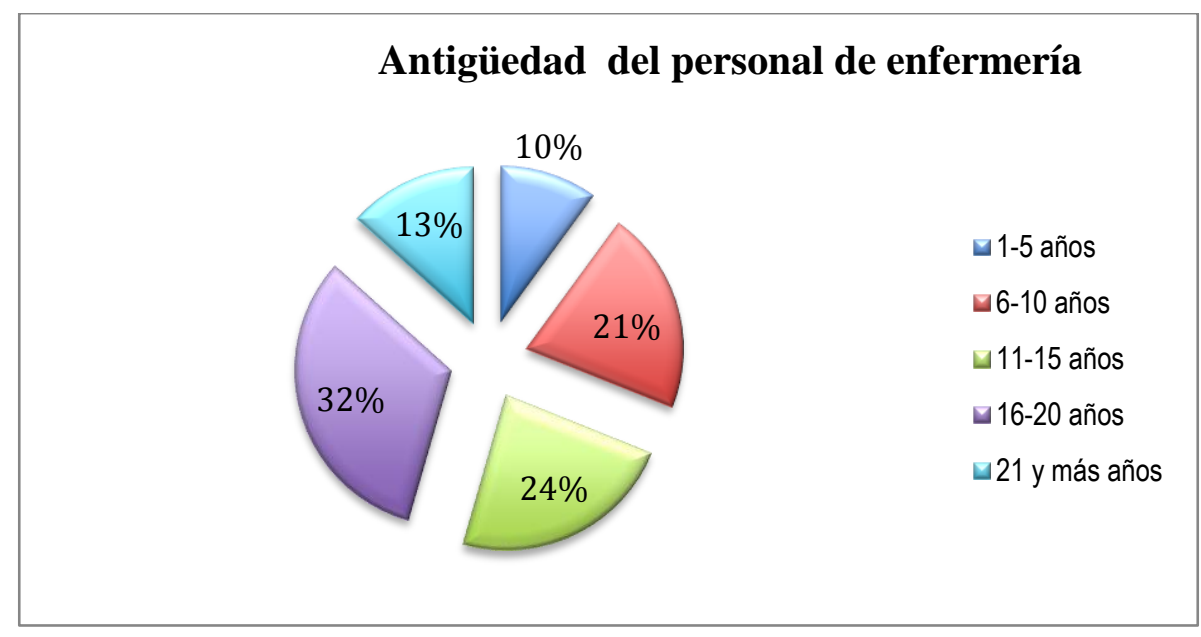

Fuente: 90 cuestionarios aplicados al personal de enfermería 


\section{Variables de satisfacción laboral del personal de enfermería}

En la satisfacción por el trabajo, $71.0 \%$ (64) de las enfermeras encuestadas dijo estar muy de acuerdo y de acuerdo con el trabajo que realiza (gráfica 8).

\section{Gráfica 8}

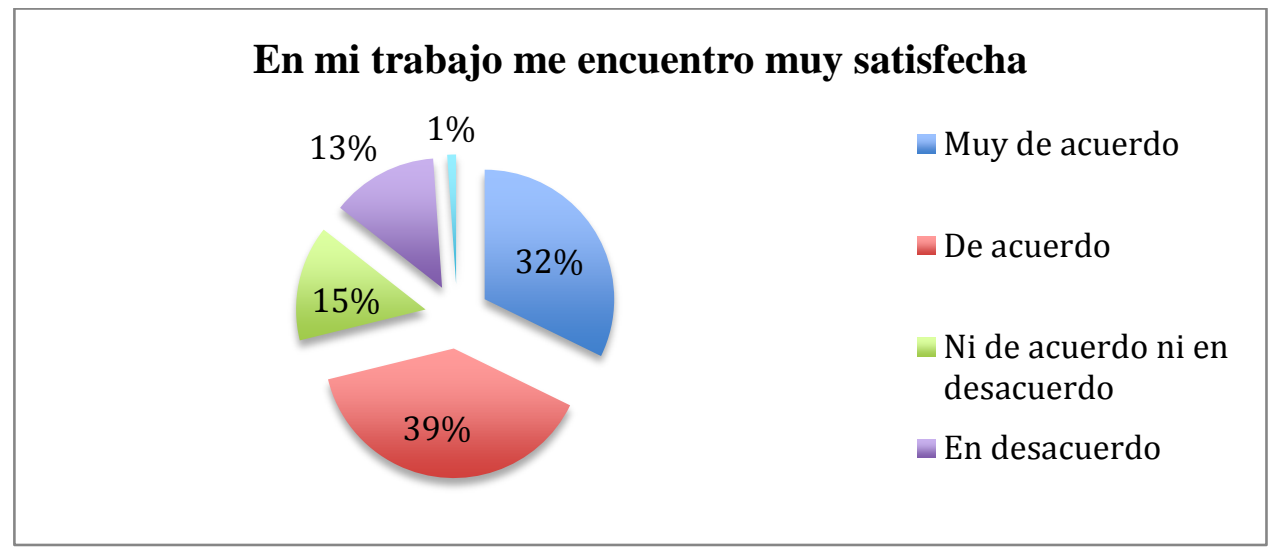

Fuente: 90 cuestionarios aplicados al personal de enfermería

Con respecto a la dimensión relación interpersonal con los jefes y la variable "ambiente tenso entre directivos y operativos de enfermería", $32.0 \%$ (29) dijo estar ni de acuerdo ni en desacuerdo, $29.0 \%$ (26) dijo estar en desacuerdo y $22.0 \%$ (20) de acuerdo, mientras que el resto eligió otras respuestas (gráfica 9).

\section{Gráfica 9}

\section{Ambiente tenso entre directivos y operativos de enfermería}

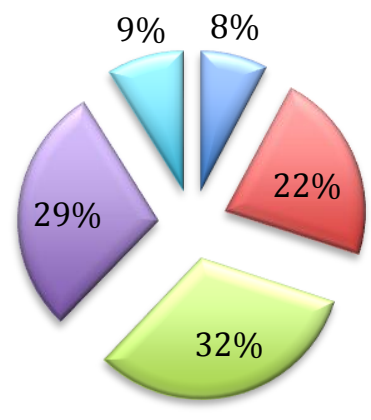

$\square$ Muy de acuerdo

$\square$ De acuerdo

$\square$ Ni de acuerdo ni en desacuerdo

$\square$ En desacuerdo

$\square$ Muy en desacuerdo

Fuente: 90 cuestionarios aplicados al personal de enfermería 
En las características extrínsecas de estatus, la pregunta fue si el sueldo de enfermería es adecuado según el trabajo que se realiza, aquí 27.0 \% (24) respondió que estaba ni de acuerdo ni en desacuerdo, $25.0 \%$ (23) dijo estar muy en desacuerdo y $21.0 \%$ (19) de acuerdo, el resto eligió otras opciones (gráfica 10).

\section{Gráfica 10}

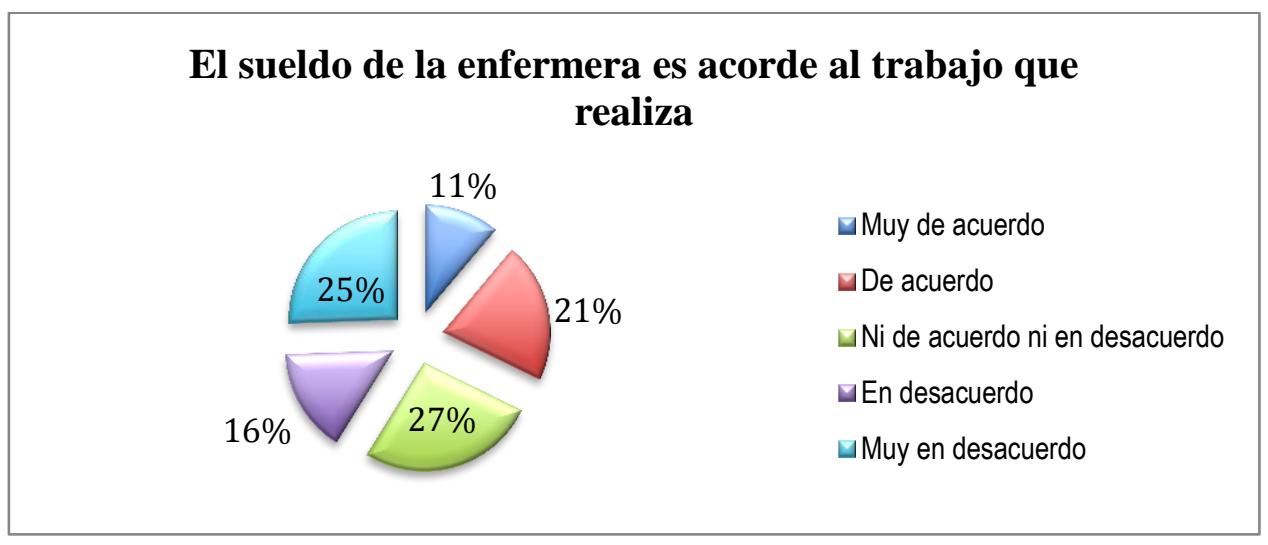

Fuente: 90 cuestionarios aplicados al personal de enfermería

En la influencia del entorno, "la insuficiencia de recursos humanos de enfermería me impide desarrollar mi trabajo", $28.0 \%$ (25) respondió estar muy de acuerdo, $27.0 \%$ (24) de acuerdo y $24.0 \%$ (22) ni de acuerdo ni en desacuerdo, el resto respondió de otra manera (gráfica 11).

\section{Gráfica 11}

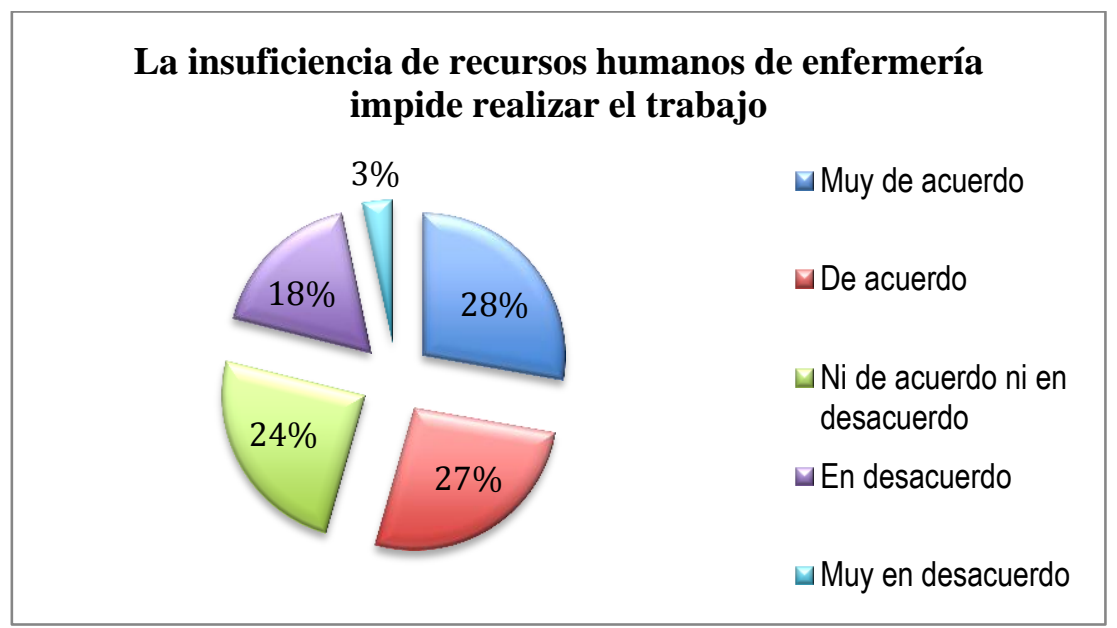

Fuente: 90 cuestionarios aplicados al personal de enfermería 
Después de aplicar la asociación de variables por medio de chi cuadrado se obtuvo la siguiente significancia estadística:

\section{Tabla 3}

\begin{tabular}{|l|l|}
\hline Asociaciones & Significancia \\
\hline $\begin{array}{l}\text { El personal de enfermería atiende las } \\
\text { necesidades físicas, psicológicas y }\end{array}$ & \\
espirituales de los pacientes vs la & .000 \\
disposición del personal de enfermería para & \\
brindarles atención durante su estancia & \\
hospitalaria. & \\
\hline $\begin{array}{l}\text { El personal de enfermería informa a los } \\
\text { pacientes sobre los cuidados o actividades }\end{array}$ & \\
a realizar vs la manera como considera las & \\
señalizaciones en el hospital: baños, & \\
escaleras, salidas de emergencia, etcétera. & .000 \\
\hline $\begin{array}{l}\text { La manera como considera los equipos que } \\
\text { utiliza el personal de enfermería para }\end{array}$ & \\
realizar su trabajo (tecnología) vs la \\
rapidez del personal de enfermería para \\
conseguir lo que necesita el paciente.
\end{tabular}




\section{Discusión}

El presente estudio tuvo como objetivo correlacionar la calidad de la atención percibida de los usuarios hospitalizados con la satisfacción de los profesionales de enfermería del Hospital General "Vasco de Quiroga" del ISSSTE en Morelia, Michoacán. Por su parte, García, M. et al. (2007) refieren que conocer las necesidades que influyen en su desempeño laboral, es una prioridad que permite a las unidades hospitalarias ejecutar estrategias que ofrezcan a las enfermeras aquellos elementos que contribuyen a mejorar su desempeño laboral, junto con una actitud de servicio que refleje la calidad de la atención y del ambiente de trabajo. Los resultados muestran que existe un mínimo porcentaje de profesionales de enfermería con insatisfacción laboral y que los pacientes perciben calidad en la atención.

De acuerdo con la definición de Moreno, A. (2005) de la calidad asistencial, esta responde de manera más adecuada a las necesidades y expectativas de las usuarias de los servicios de salud, con los recursos humanos y materiales de los que disponen y el nivel de desarrollo científico actual, lo cual les permite alcanzar la máxima satisfacción al costo más razonable. Se observó que se dieron respuesta a las necesidades e intereses de los pacientes con los recursos humanos (personal de enfermería) y materiales (equipamiento), y que existe un alto porcentaje de profesionales de enfermería satisfechos laboralmente.

Bravo, Peiró y Rodríguez (Berrios, P. et al., 2006, p. 5) refieren que algunos de los aspectos de satisfacción laboral son: la empresa, los compañeros de trabajo, las condiciones de trabajo, el progreso en la carrera, las perspectivas de promoción, el sueldo, los subordinados, la estabilidad en el trabajo, el tipo de trabajo, la cantidad de trabajo, el desarrollo personal, la satisfacción extrínseca y la satisfacción intrínseca generales. Todo ello concuerda con los resultados que se obtuvieron en esta investigación, donde la mayoría de las enfermeras refirieron estar satisfechas en algunos de los factores extrínsecos e intrínsecos.

\section{Conclusiones}

Más de la mitad de los pacientes objeto de estudio tienen de 30 a 40 y tantos años de edad, la mayoría cursó la educación básica y algunos el nivel superior. Las tres cuartas partes de los entrevistados pertenecen al turno matutino y más de la mitad lleva de uno a tres días 
hospitalizado, tiempo suficiente para que puedan valorar la calidad de la atención en enfermería que reciben.

Con respecto a los factores tangibles, los usuarios manifestaron que la tecnología, el equipamiento, las señalizaciones, el estado de las habitaciones y la apariencia del personal de enfermería fueron mucho mejores de lo que esperaban, es decir, que el hospital cuenta con todo lo necesario para brindar atención.

En cuanto a los factores intangibles, el paciente dijo que estaba mejor de lo que esperaba y mucho mejor de lo que esperaba, así como que los profesionales de enfermería se interesan en que su estancia sea agradable, reciba información sobre los cuidados o actividades que le van a realizar, solucionar las problemáticas de su competencia, inspirar confianza y ser amables. También opinó que cuentan con la preparación académica necesaria, que su trato fue mucho mejor de lo que esperaba y que comprenden sus necesidades físicas, psicológicas y espirituales.

Con respecto a los profesionales de enfermería, se observó que casi la mitad tiene cuarenta o más años de edad, 7 de cada 10 son licenciadas en enfermería, casi la mitad forma parte del personal de base y está contratada con la categoría de enfermera general y tiene más de 16 años de antigüedad. Siete de cada diez enfermeras refirió que está muy de acuerdo y de acuerdo con su trabajo, 2 de cada 10 dijo que está de acuerdo en que su trabajo no es reconocido, 3 de cada 10 padece estrés laboral, y la minoría comentó que siente no estar capacitada para el trabajo. Un tercio mencionó que el exceso de trabajo no le permite realizar sus actividades con calidad, y un considerable número precisó que tiene poca oportunidad de aprender a hacer cosas nuevas relacionadas con su profesión. El ambiente laboral entre los jefes y los subordinados lo perciben algo tenso, 3 de cada 10 refirió que el salario que perciben no va de acuerdo a su carga laboral. Por otro lado, algunas enfermeras dijeron que no perciben monotonía laboral, mientras que cierto grupo no realiza rotación de servicios debido a su edad, antigüedad, problemas de salud u otros motivos. Finalmente, la insuficiencia de recursos humanos les impide desarrollar su trabajo de manera adecuada.

Todo lo anterior puede conducirnos a afirmar que algunas enfermeras padecen insatisfacción laboral, a pesar de lo cual el paciente hospitalizado sigue percibiendo calidad en el cuidado que recibe. 


\section{Bibliografía}

Barragán, J y Manrique, Fred. ( 2010). Validez y confiabilidad del Servqhos para enfermería en $\begin{array}{lllll}\text { Boyacá, } & \text { Colombia, } & \text { (2). } & \text { Recuperado }\end{array}$ http://www.revistas.unal.edu.co/index.php/avenferm/article/view/21376/22431

Barragán JA, Pedraza YD, Rincón MR. (2013). Calidad percibida Calidad percibida de la atención de enfermería por usuarios hospitalizados, 31(2), 187-193 . Recuperado de http://www.scielo.org.co/pdf/rfnsp/v31n2/v31n2a04.pdf

Berrios, M. P, Agusto, J.M y Aguilar, M.C. (2006). Inteligencia emocional percibida y satisfacción laboral en contextos hospitalarios. Un estudio exploratorio con profesionales de enfermería. Index Enferm, 15 (54). Recuperado de http://scielo.isciii.es/scielo.php?script=sci_arttext\&pid=S1132-12962006002200006

Borré, M, Lenis, C y González G. (2014). Utilidad del cuestionario SERVQHOS-E para medir calidad percibida de la atención de enfermería. CES Salud Pública, 5 (2), 127-136. Recuperado dehttps://www.google.com.mx/search?client=safari\&rls=en\&q=Utilidad+del+cuestionari o+SERVQHOSE+para+medir+calidad+percibida+de+la+atencio\% $\%$ C $\% 1 \mathrm{n}+$ de+enferme $\underline{\text { ri\%CC\%81a\&ie=UTF-8\&oe=UTF-8\&gfe_rd=cr\&ei=9 1V9i_LMnCqAXz5aO4Aw }}$

Borré, Y. M. y Vega, Y. (2014). Calidad percibida de la atención de enfermería por pacientes hospitalizados.Ciencia y Enfermería, XX (3), 81-94. Recuperado de http://www.scielo.cl/pdf/cienf/v20n3/art_08.pdf.

De Andrade, S; Torres, B; Ocampo, G y Alcalá E (2012). Teoría de la Motivación-Higiene de Herzberg. Universidad Simón Bolívar de Caracas. Recupeado de http://prof.usb.ve/lcolmen/Trabajo-Grupo1-seccion-02.pdf

Hernández, A y Zarate R.M.(2011). Hospital magnético: Escenario ideal que garantiza calidad del cuidado y la satisfacción laboral en enfermería. Revista EnfermeríaUniversitaria ENEO-UNAM, 8 (2), 25-32. Recuperado de http://www.medigraphic.com/pdfs/enfuni/eu2011/eu112e.pdf 
García, M, Lujan, M.E y Martínez, M.A (2007) . Satisfacción laboral del personal de salud [ versión electrónica] Rev Enferm Inst Mex Seguro Social,15 (2), 63. Recuperado de http://www.medigraphic.com/pdfs/enfermeriaimss/eim-2007/eim072b.pdf

Lenis, V, Manrique, Ab y Fred, G.(2015). Calidad del cuidado de enfermería percibida por pacientes hospitalizados en una clínica privada de Barranquilla; Aquichan,15 (3): 413 425. DOI: 10.5294/aqui.2015.15.3.9 Recuperado de http://www.scielo.org.co/scielo.php?script=sci_abstract\&pid=S165759972015000300009

Massip, C, Ortiz, Rosa M.; Llantá, M.C, Peña, M, Infante, Idalmis. (2008). La evaluación de la satisfacción en salud: un reto a la calidad. Revista Cubana de Salud Pública, 34, (4) 1-10 .Recuperado desde http://www.redalyc.org/articulo.oa?id=21419854013

Moreno, A ( 2005). La calidad de la acción de enfermería. Enfermería Global, 6 (1),2.Recuperado de http://revistas.um.es/eglobal/article/viewFile/522/536

Navarro, E, Linares y Montana, A. (2010).Factores de Satisfacción Laboral Evocados por los Profesionales de la Construcción en la Comunidad Valenciana (España). Revista de la Construcción, $\quad 9(1), \quad 4-19 . \quad$ Recuperado de http://www.scielo.cl/scielo.php?script=sci_arttext\&pid=S0718-915X20100001000

Net, À y Suñol, $\mathrm{R}$ (s/f). La calidad de la atencion. Recuperado de http://www.coordinadoraprofunds.org/docs/214/rosa_sunol.pdf

Nuñez, E, Estéves, G, Hernández, P y Marrero, C. (2007). Una propuesta destinada a complementar el cuestionario Font-Roja de satisfacción laboral. Gac Sanit.,21(2):136-41. Recuperado de http://www.scielosp.org/scielo.php?script=sci_arttext\&pid=S021391112007000200008

Racoveanu, N. T. y Staehr, K.(1995). Tecnologia para el mejoramiento continuode Ia calidad de Ia atencion sanitaria. Foro Mundial de Salud, 16, 158. Recuperado de http://apps.who.int/iris/bitstream/10665/53791/1/WHF_1995_16_n2_p158-165_spa.pdf 
Racoveanu, N. T. y Staehr, K. (2013) Calidad de la atención.Tecnologia para el mejoramiento continuo de Ia calidad de Ia atencion sanitaria. Foro Mundial de Ia Salud (16), Recuperado de http://apps.who.int/iris/bitstream/10665/53791/1/WHF_1995_16_n2_p158-165_spa.pdf

Tapia, H, Ramírez, C e islas, E. (2009). Satisfacción laboral en enfermeras del hospital de oncología Centro Medico Nacional siglo XXI IMSS, Revista Enfermería Universitaria ENEO-UNAM . 6. (4), 22. Recuperado de http://www.medigraphic.com/pdfs/enfuni/eu2009/eu094d.pdf 